\title{
AIDEZ ANDROLOGIE
}

ANDROLOGIE aura bientôt 2 ans. Malgré les nombreuses difficultés matérielles qui n'ont pas manqué de se dresser, le journal aura tenu son pari de publier chaque année une quarantaine d'articles scientifiques de bonne tenue, tout en continuant de servir de trait d'union aux membres de la Société d'Andrologie de Langue Française. Il est maintenant répertorié par plusieurs index bibliographiques, et en passe de l'être par d'autres.

Ce but n'aurait pu être atteint sans l'aide efficace du comité de rédaction qu'il faut remercier ici. Je veux tout particulièrement citer André Demoulin et André Clavert, Michèle Buvat-Herbaut pour son inlassable travail d'édition, et David Mortimer qui a montré depuis Sydney une extraordinaire disponibilité pour réviser nos résumés anglais. Il faut aussi vivement remercier les professionnels de l'industrie pharmaceutique dont le soutien matériel a assuré une grande part des frais d'édition du journal.

Que nous ayions voulu faire d'ANDROLOGIE un journal scientifique plutôt qu'une revue de vulgarisation fait qu'il s'adresse à un public plus limité et encore incomplètement ciblé. Ce journal reste aujourd'hui très fragile et a besoin de votre aide, non seulement pour croitre, mais tout simplement pour survivre. Ceux qui souhaitent que se poursuive cette expérience originale d'une publication scientifique très spécialisée en langue française doivent se mobiliser, et la soutenir de l'une ou de plusieurs des façons suivantes :

- en lui envoyant des articles scientifiques, ou en suscitant de tels envois

- en envoyant des lettres à l'éditeur, ou des annonces importantes pour l'Andrologie ;

- en suscitant des abonnements, particulièrement de leurs bibliothèques, pour faciliter la diffusion du journal ;

- en citant en référence les articles publiés dans ANDROLOGIE;

- pour ce qui concerne les enseignants, en portant à la connaissance de leurs étudiants l'existence du journal.

Soyez convaincus que l'avenir d'ANDROLOGIE repose en grande part dans vos mains. 